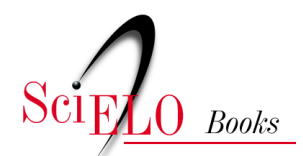

\title{
Expresiones diacrónicas de subjetividad en el español andino peruano
}

\author{
Anna María Escobar \\ Claudia Crespo
}

\section{SciELO Books / SciELO Livros / SciELO Libros}

ESCOBAR, A. M., and CRESPO, C. Expresiones diacrónicas de subjetividad en el español andino peruano. in: HABOUD BUMACHAR, M., SÁNCHEZ AVENDAÑO, C., and GARCÉS VELÁSQUEZ, F., eds. Desplazamiento lingüístico y revitalización: reflexiones y metodologías emergentes [online]. Quito: Editorial Abya-Yala, 2020, pp. 293-316. Desafíos en la Diversidad collection, n. 2. ISBN: 978-9978-10-541-2. http://doi.org/10.7476/9789978105726.0013.

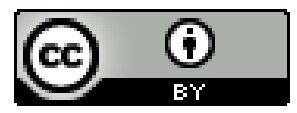

All the contents of this work, except where otherwise noted, is licensed under a Creative Commons Attribution 4.0 International license.

Todo o conteúdo deste trabalho, exceto quando houver ressalva, é publicado sob a licença Creative Commons Atribição 4.0.

Todo el contenido de esta obra, excepto donde se indique lo contrario, está bajo licencia de la licencia Creative Commons Reconocimento 4.0. 


\title{
Expresiones diacrónicas de subjetividad en el español andino peruano
}

\author{
Anna María Escobar ${ }^{1}$ \\ Claudia Crespo ${ }^{2}$
}

\begin{abstract}
Resumen
Este trabajo se enfoca en variedades del español andino que se emplean en el Perú. Se documenta el análisis de influencias semánticas del quechua en variedades de la región. Específicamente, se indaga sobre el concepto de subjetividad que expresa el pretérito perfecto compuesto (PP) en variedades del español andino. Nos centramos en la variedad peruana en la cual la subjetividad se expresa a través de la función evidencial de conocimiento directo. Mediante el contraste del análisis de datos dialectales peruanos sincrónicos (del español andino y el español ribereño) y diacrónicos (de los sesenta, ochenta y 2015), emergen en el análisis una serie de factores lingüísticos que favorecen la expresión de subjetividad. Se propone que estos factores lingüísticos que favorecen el uso del PP evidencial están conectados al hablante-emisor (primera persona gramatical, presencia gramatical del hablante-emisor en la oración, rol sintáctico del hablante como sujeto, rol semántico del hablante como no agente) y al contexto tempoaspectual (presencia de expresión temporal de presente, referencia temporal indeterminada, Aktionsart del verbo como no marcado). Se arguye que el favorecimiento semántico-gramatical hacia el hablante y hacia una temporalidad indeterminada actúan en conjunto dando una lectura subjetiva de la función evidencial de conocimiento directo; función que representa una etapa de gramaticalización más avanzada del PP en esta variedad andina peruana. Futuros estudios comparativos con otras variedades del español andino ayudarán a refinar esta propuesta para el contacto del español con lenguas andinas y otras lenguas amerindias.
\end{abstract}

\section{Introducción}

El análisis de la expresión de 'subjetividad' en la lengua es un tema bastante discutido en la literatura lingüística. Si bien la subjetivización se describe como un proceso semántico y pragmático (Traugott, 1995, p. 36; 2003, 2010) que abarca lo lexical y lo grama-

\footnotetext{
1 University of Illinois at Urbana-Champaign.

2 Pontificia Universidad Católica del Perú.
} 
tical, el ámbito gramatical, que es el foco de este trabajo, es menos estudiado en la lingüística hispánica. Sin embargo, su importancia es especialmente relevante en el estudio del contacto entre el español con una lengua indígena, especialmente el quechua, que contiene contrastes gramaticales relacionados a la evidencialidad. Es decir, nos referimos al uso de marcadores gramaticales encontrados en el quechua que expresan si la información transmitida por el hablante es de conocimiento directo o indirecto. Nos centramos aquí en la expresión de subjetividad en el llamado pretérito perfecto compuesto (PP) del español andino peruano que tiene función evidencial de conocimiento directo (Escobar \& Crespo 2020). El objetivo de este trabajo es indagar sobre los factores lingüísticos que ayudan a definir esta función evidencial del PP peruano a partir de una profundización del concepto de 'subjetividad'.

La evidencialidad es una categoría semántico-pragmática (Andersen, 1986; cf. Chafe \& Nichols, 1986; Aikhenvald, 2004) que está al centro del presente análisis en su versión de evidencia directa. La evidencialidad marca gramaticalmente la 'fuente de la información que transmite el hablante' y desde la perspectiva del hablante (Chafe \& Nichols, 1986; Willett, 1988; Aikhenvald, 2004, p. 3; Nucholls \& Michael, 2014). Es decir, expresa si la fuente de la información es por experiencia directa del hablante o por experiencia indirecta. La subjetividad, por tanto, está presente en el ámbito de la evidencialidad en aquellos contextos que expresan conocimiento directo y desde la experiencia del hablante (cf. Chafe \& Nichols, 1986; Anderson, 1986; Bergqvist, 2017).

En la Figura 1 presentamos las distinciones que Willett (1988) encontró en su estudio sobre la evidencialidad en las lenguas del mundo: 


\section{5}

Figura 1

Tipos de fuentes de la información

\begin{tabular}{|c|c|l|l|l|l|l|l|}
\hline \multicolumn{5}{|c|}{ Tipos de fuente del conocimiento de la información transmitida } \\
\hline \multicolumn{3}{|c|}{ Directa } & \multicolumn{5}{c|}{ Indirecta } \\
\hline \multicolumn{3}{|c|}{ Atestada } & \multicolumn{3}{c|}{ Reportada } & \multicolumn{2}{c|}{ Inferencia } \\
\hline visual & auditiva & $\begin{array}{l}\text { otros } \\
\text { sentidos }\end{array}$ & $2^{\text {da }}$ mano & $3^{\text {ra } \text { mano }}$ & folklor & resultados & inferencia \\
\hline
\end{tabular}

La evidencialidad se ha estudiado en el español particularmente en casos de información obtenida indirectamente (o reportadas) y expresada lexicalmente (e.g., Cornillie, 2007; Hennemann, 2012; Hassler, 2015; Rodríguez Ramalle, 2015). En nuestro trabajo, nos enfocamos, en cambio, en la expresión gramatical de la experiencia directa $y$, por lo tanto, nos centramos en develar los factores lingüísticos que nos puedan ayudar a entender mejor la 'perspectiva del hablante' en el uso del PP-evidencial peruano. Nuestro fin es descubrir los contextos lingüísticos en los que el PP con información directa (o vivida por el mismo hablante) es favorecido en esta variedad de español en contacto.

Mediante el análisis de datos diacrónicos de dos variedades peruanas, nos embarcamos en la delineación de factores lingüísticos que nos permitan estudiar la 'subjetividad'. Partimos de un entender el 'conocimiento directo' como haciendo referencia al ámbito de la experiencia del hablante (cf. Chafe \& Nichols, 1986; Anderson, 1986; Bergqvist, 2017). Ilustramos esta función en el PP-evidencial con los ejemplos en (1), donde presentamos una serie de lecturas que se pueden dar en diferentes variedades de español a la oración (el gallo) me ha pateado mi ojo.

1. (El gallo) me ha pateado mi ojo.

a. el 'patear' acaba de ocurrirle recientemente a la persona que lo dijo, por lo que el 'patear' se puede entender como que le ocurrió al hablante en el mismo día. 


\section{6}

b. el 'patear' ocurrió ayer o en la misma semana o antes, pero todavía hay marcas físicas del evento.

c. el 'patear' pudo haber ocurrido en el pasado cuando la persona era muy joven, pero la persona que habla se acuerda de este evento que vivió. No se lo han contado.

En la lectura de (1a), se interpreta el enunciado como que la persona a quien le ocurrió 'el patear' todavía está llorando o está en shock y/o tiene heridas frescas en el ojo o en la cara. Esta interpretación atribuye a la distancia temporal entre el 'patear' y la enunciación como corta, lo que lleva a que el oyente entienda que el evento de 'patear' debe de haber ocurrido muy recientemente al momento de la enunciación, ya sea en el día o incluso en la misma tarde. En otras variedades de español, las lecturas pueden ser otras. Por ejemplo, en la lectura en (1b), el oyente no considera que el evento tuviera lugar en el mismo día de la enunciación. En cambio, interpreta que este evento pudo haber tenido lugar el día anterior, o en la misma semana, o incluso en el mismo mes. En cualquier caso, se entiende que hay evidencia física visible que remite al evento original del 'patear'. En la lectura en (1c), en cambio, del español andino peruano, 'el patear' puede haber ocurrido en la niñez del hablante adulto, en cualquier momento de su pasado (como es el caso en este ejemplo, de nuestros datos peruanos). Lo importante aquí es que la participante se acuerda de lo que le ocurrió (no se lo contaron) y lo narra en el contexto de explicar su uso de anteojos.

Este uso evidencial del PP para hacer referencia a experiencias vividas por el narrador/hablante se ha reportado en el español andino peruano en varios estudios (Schumacher, 1975, 1980; Escobar, 1993, 1994, 1997; Klee \& Ocampo, 1995; Sánchez, 2004; Jara Yupanqui, 2006, 2013; Howe, 2006, 2013; Howe \& Schwenter, 2008; Rodríguez Louro \& Jara Yupanqui, 2011; Álvarez Garriga, 


\section{7}

2012; García Tesoro \& Yang, 2018), así como en el español boliviano (Mendoza, 1991, 1992; Stratford, 1991; Babel, 2009). En la variedad andina ecuatoriana, se encuentra un uso evidencial del PP que corresponde, en cambio, al reportativo (Bustamante, 1991; Dumont, 2013; Pfänder \& Palacios, 2013), así como una preferencia por formas perifrásticas con funciones tempo-aspectuales (cf. Haboud, 1998). De tal manera, mientras en las variedades peruanas y bolivianas el PP de evidencia directa contrasta con el pluscuamperfecto que tiene la función de reportativo o evidencia indirecta, en las variedades ecuatorianas el PP tiene función de reportativo y contrasta con el pretérito que tiene la función de evidencia directa.

La presencia del español y las lenguas andinas en la misma macrorregión andina ha llevado a un contacto lingüístico y cultural entre lenguas que no están relacionadas ni estructural ni tipológicamente (flexiva <> aglutinante; $\mathrm{VO}<>\mathrm{OV}$ ), ni ontológicamente (antropocéntrica y egocéntrica $<>$ alocéntrica y narrativa, cf. Shapero 2017; Mannheim, en prensa). Además, en las lenguas andinas, incluyendo el quechua, el aimara y el jaqaru (Weber, 1986; Hardman, 1981, 1986), se encuentran expresiones gramaticales (sufijos) con función evidencial, mientras en el español la evidencialidad se expresa primordialmente en el ámbito léxico (cf. Henemann, 2012; De la Mora \& Maldonado, 2015; Cornillie, 2017).

Efectivamente, la evidencialidad se expresa en las lenguas andinas a través de sufijos discursivos que, según la variedad de quechua del que se trate, diferencian al menos tres funciones en las variedades peruanas (cf. Weber, 1986; Cerrón Palomino, 1987; Floyd, 1999; Faller, 2002, 2011; Hintz, 2007; Nuckolls, 2014). Se diferencian (1) el conocimiento directo de primera mano, con -mi (traducción aproximada: 'yo sé que... porque lo he vivido'); así como dos distinciones adicionales de conocimiento indirecto, (2) el conocimiento 
reportado con -si (traducción aproximada: 'alguien me dijo que...') y (3) una inferencia o conjetura concha (traducción aproximada: 'me parece/ yo creo/pienso que...').

En la lingüística hispánica se ha dado especial atención al estudio de la información reportada, tanto en variedades diacrónicas del español, como en variedades modernas. El foco primordial ha sido en la expresión dizque (cf. Bol, Babel 2009; Alcázar, 2018), con función reportativa. De la Mora y Maldonado (2015) arguyen que en diferentes variedades de español esta expresión se ha gramaticalizado a un marcador pragmático. Otros estudios se han centrado en el uso de dice, también del verbo decir, especialmente en variedades del español en contacto con el quechua (Andrade, 2007), y también en la combinación de dice con el verbo que le sigue en pluscuamperfecto (Schumacher, 1980; Escobar, 1994; Klee \& Ocampo, 1995). Finalmente, Demonte y Fernández Soriano (2013) estudian la expresión que sola para introducir información reportada.

En nuestro trabajo, presentamos análisis de datos peruanos de los siglos XX y XXI para centrarnos en el uso evidencial del PP en variedades andinas y limeñas, considerando que la capital Lima es un espacio donde existe un intenso contacto dialectal entre diferentes variedades peruanas, con especial presencia de variedades andinas (Escobar, 2014). El objetivo es develar qué factores lingüísticos y gramaticales, representados en el discurso, contribuyen a favorecer una lectura de conocimiento directo, por la cual el PP expresa información que es 'real' para el hablante, porque es información de primera mano (Chafe \& Nichols, 1986, p. vii). Argüimos que definir los factores lingüísticos y gramaticales expresados en el contexto del uso del PP son relevantes para el estudio de la subjetividad y la evidencialidad en el español andino. 


\section{9}

\section{Conocimiento directo}

Para poder proponer qué factores lingüísticos podrían ser relevantes empezamos tratando de explorar el concepto de conocimiento directo. La literatura lingüística define el 'conocimiento directo' como conectado al discurso, en el sentido que expresa el 'punto de vista del hablante' (Bergqvist, 2017) comunicando 'información que es verdadera para el hablante' (Chafe \& Nichols, 1986, p. vii). Hassler agrega que se trata de un 'posicionamiento del hablante' (2015), que es descrito por otros como un 'mayor grado de compromiso del hablante' con lo que dice y en oposición a la experiencia personal indirecta (cf. Cornillie 2017). Desde una perspectiva diferente, algunos autores enfatizan que la evidencialidad, como concepto gramatical, está conectada con la 'deixis' (cf. Boye, 2018). En otro trabajo, proponemos que la emergencia del PP-evidencial del español andino (con datos peruanos) está conectada tanto a una reinterpretación del concepto de 'relevancia con el presente', que es parte de la evolución de la forma verbal PP, así como conectada a la perspectiva del hablante y desde el conocimiento del hablante (Escobar \& Crespo, 2020). La gramaticalización del PP-evidencial peruano representaría un cambio semántico que va desde expresar un grado de distancia temporal con un evento pasado a expresar que un evento pasado al que se alude es de conocimiento directo del hablante.

Si el 'conocimiento directo' está conectado a la perspectiva del hablante y a su experiencia, entonces los factores lingüísticos que definen la subjetividad (y que deben de aparecer con el PPevidencial peruano) deben incluir los siguientes cuatro factores. El primero trata de la persona gramatical del sujeto, expresada en la primera persona singular (1s). Esta favorecería la expresión de la perspectiva del hablante y, por tanto, favorecería el uso del PP- 
300

evidencial. La expresión del pronombre sujeto de 1s (en oposición a su omisión, fenómeno más común en las variedades peruanas, Cerrón Palomino, 2018) resaltaría aun más la primera persona gramatical y, por extensión, el hablante. Por tanto, la expresión del pronombre 1s sería otro factor relevante (Davidson, 1996). Si bien se esperaría igualmente que el rol sintáctico del hablante sea el de sujeto, el rol semántico del hablante-emisor (que es sujeto) debería ser el de [- agente], ya que el PP debería estar favorecido con verbos cognitivos (pensar, querer), de percepción (ver, oir) y psicológicos (asustarse, preocuparse), todos enfocados en el hablante y en un sujeto experimentante, de acuerdo con Van Valin y LaPolla (1997).

Los estudios sobre el PP en la lingüística tienden a centrarse no en el hablante o el sujeto, como en nuestro caso, sino en la temporalidad que define la evolución del PP en las lenguas europeas (español, francés, italiano, alemán, inglés). En un estudio que contrasta la gramaticalización del PP en el español mexicano y el español peninsular (de Alicante), Schwenter y Torres Cacoullos (2008) proponen que el factor lingüístico que cumple la función de señalar si la gramaticalización del PP avanza hacia un perfectivo, desde un presente perfecto (o anterior, Bybee et al., 1994) que marca relevancia con el presente hasta un perfectivo, estaría en manos de expresiones temporales que marcan el presente. Cuando estas referencias al tiempo presente se debilitan, la referencia temporal se vuelve cada vez más indeterminada, lo que favorecería la gramaticalización hacia el perfectivo. Si un avance en el proceso de gramaticalización se marca con la temporalidad indeterminada, entonces el PP-evidencial debería ser favorecido por la indeterminación temporal, si bien su mayor gramaticalización no lo lleva a la perfectividad. Bybee et al. (1994, cap. 3) encuentran que hay lenguas en el mundo que toman otro camino en su gramaticali- 


\section{1}

zación, como parece ser el caso del español andino peruano. La indeterminación temporal no haría referencia, en este caso, a la no marcación abierta de un referente temporal específico, sino a una referencia temporal vaga que no sitúa el evento en un momento temporal específico. En relación con la temporalidad, también debemos considerar la semántica lexical vendleriana (o Aktionsart) del verbo. El durativo (correr, hablar) y el estado (vivir, estar), que no expresan un límite temporal, deberían favorecer más el PP-evidencial que el durativo-télico (buscar, construir) o el propiamente télico (romperse, llegar) que sí expresan algún límite del evento.

Finalmente, los estudios lingüísticos también proponen que el 'conocimiento directo' se conecta con la deixis (cf. Boye, 2018). Por lo tanto, si el foco es en el hablante y su mundo, entonces, si el hablante no es sujeto del enunciado, este debe de estar presente en otra función sintáctica en el discurso y su presencia debería ser más alta que su ausencia.

A partir de esta base teórica, presentamos los siete factores lingüísticos descritos como expresiones gramaticales que favorecerán el uso del PP-evidencial, según los argumentos antes desarrollados. A continuación, pasamos a analizar los casos del PP encontrados en los datos. Se trata de un total de 690 casos en narraciones de 24 hablantes que representan cuatro variedades de español peruano que se diferencian diacrónica y dialectalmente.

\section{Análisis y resultados}

Usamos cuatro corpora del español peruano que representan tres momentos diacrónicos (los sesenta, los ochenta y el 2015) y dos variedades dialectales diferentes (andina y limeña). Los primeros dos corpora, que llamamos Andino-60 y Andino-80, provienen de ha- 
blantes de la región centro-sur del Perú, donde se encuentra el mayor número de hablantes del quechua, y representan los sesenta y los ochenta del siglo pasado. Incluyen grabaciones de doce individuos (divididos igualmente) que crecieron expuestos y hablando el español y el quechua desde la niñez (2L1). Las grabaciones contienen historias personales.

El tercer y el cuarto corpora provienen de Lima. El primer corpus limeño al que llamamos Lima-80 son historias personales de seis hablantes limeños nacidos y crecidos en la capital, con padres y abuelos nacidos en Lima también y tomados de Caravedo (1989, 1990). El segundo corpus limeño, que llamamos Lima-15, incluye seis grabaciones del corpus recogido por el proyecto CLoTILdE (Contacto Lingüistico o Trayectorias de Influencia Lingüistica en variedades del Español peruano, dirigido por la primera autora) en el 2015, de personas que representan la primera generación nacida en Lima, de padres migrantes de otras regiones del país. En vista de que la población actual limeña con tres generaciones nacidas en Lima solo representa el $12 \%$ de la población de la capital peruana (Arellano \& Burgos, 2010), este corpus representa la nueva norma limeña, ya que los dos corpora de Lima (el del 80 y el 15) provienen de individuos con educación superior.

El Cuadro 1 muestra cómo la primera persona singular es favorecida especialmente en las dos variedades limeñas. Los datos diferentes del Andino-60 y el Andino-80 sugieren que sería de interés mirar a más datos de la variedad andina del 80 para verificar si los resultados aquí presentados son representativos, ya que muestran una tendencia diferente al de las otras variedades.

La preferencia por la primera persona en los datos limeños va acompañada de un alto porcentaje de pronombre sujeto expresado. 
El valor de $54 \%$ de pronombres expresos en los datos es incluso más alto que los porcentajes de pronombres expresos encontrados en variedades caribeñas en la literatura. Incluso, este porcentaje contrasta altamente con los valores encontrados en las variedades andinas peruanas anteriormente estudiadas (15.1\%, Cerrón Palomino, 2018).

\section{Cuadro 1}

\section{La persona gramatical de primera persona} en las variedades peruanas

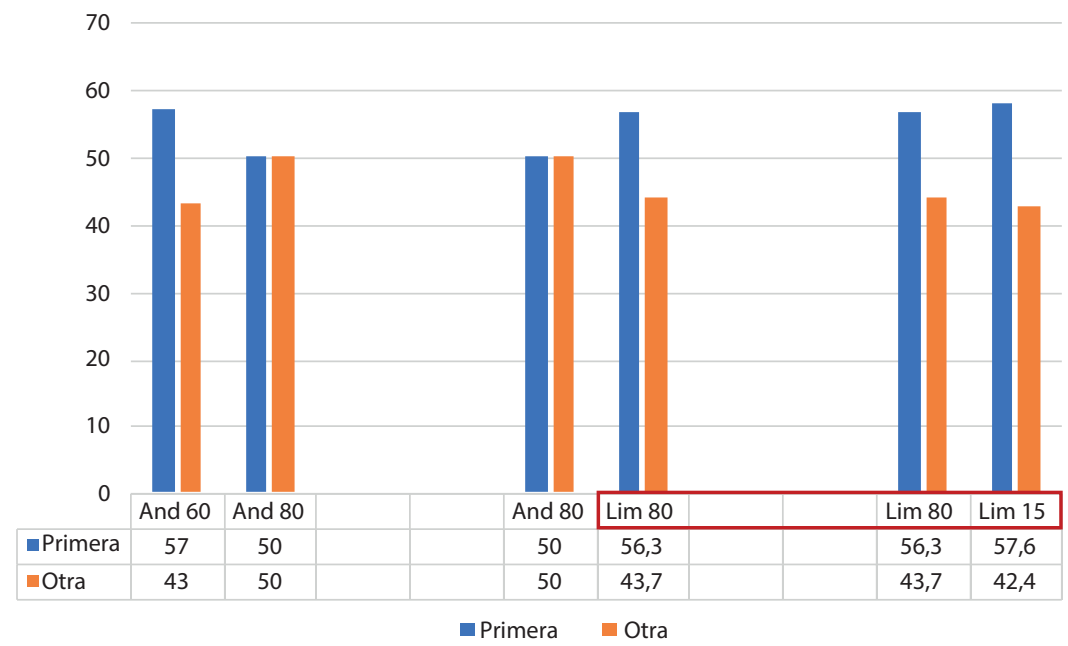

La presencia del hablante-emisor dentro del enunciado en alguna función sintáctica ocurre en el 70\% o más en todas las variedades estudiadas del español peruano (Cuadro 2). Este resultado comprueba que la función del PP en estas variedades del español está conectada al concepto de subjetividad. 


\section{Cuadro 2}

La presencia del hablante-emisor en el enunciado en las variedades peruanas

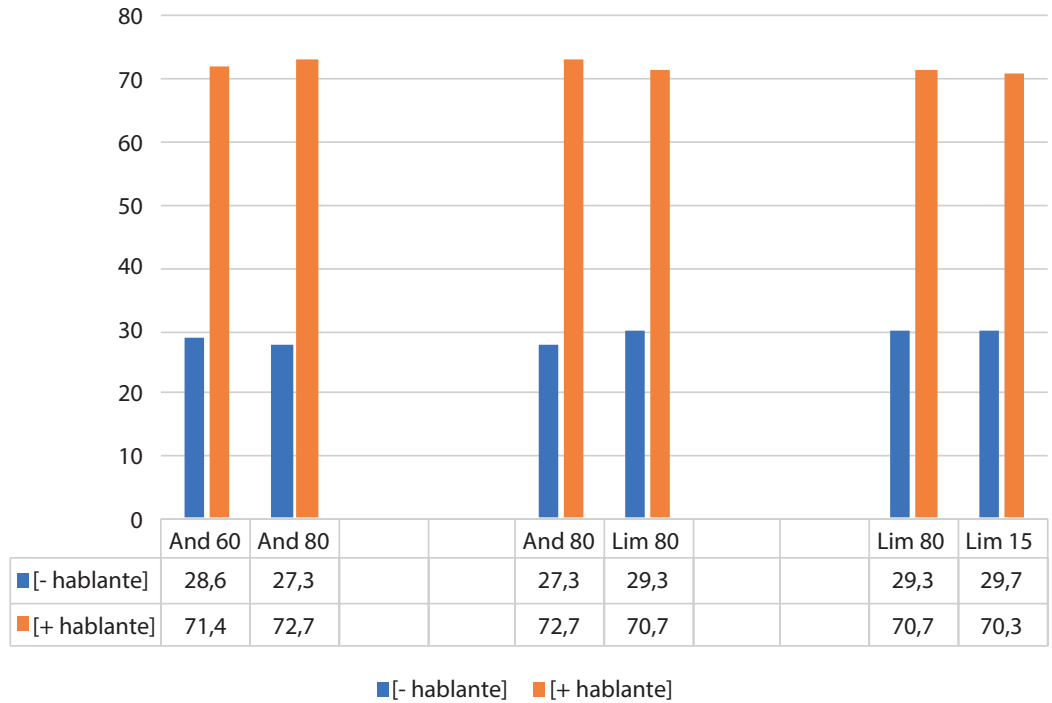

Cuando el hablante tiene el rol sintáctico de sujeto del enunciado, el porcentaje es altísimo comparado con la presencia del hablante-emisor como objeto en el enunciado (Cuadro 3). Tanto la primera persona gramatical como su función como sujeto del enunciado refuerzan la subjetividad asignada a la forma verbal PP y se encuentra en todas las variedades del español peruano estudiadas. Incluso, la presencia del hablante en alguna función sintáctica del enunciado corrobora la fuerte conexión entre el PP y la representación del hablante en el enunciado. 
305

\section{Cuadro 3}

El rol sintáctico del hablante-emisor en las variedades peruanas

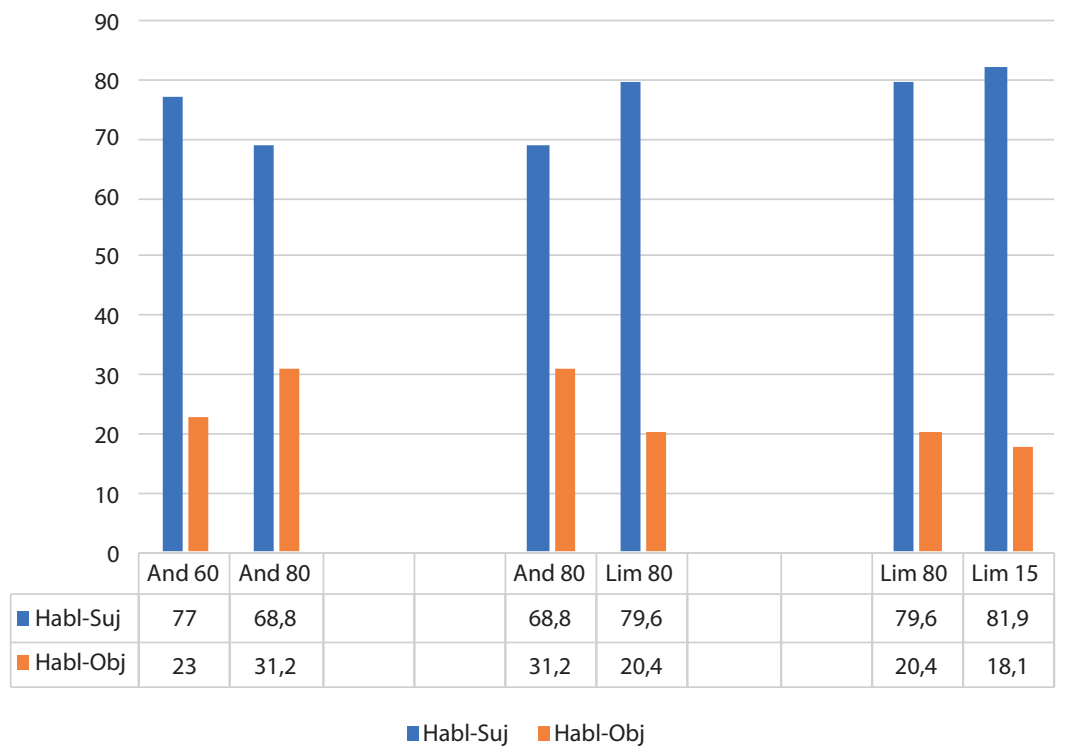

El rol semántico del hablante cuando es sujeto del enunciado es más alto cuando el sujeto expresa roles no agentivos. La no agentividad es favorecida mediante el uso de verbos cognitivos, de percepción y de experiencia que además expresan la perspectiva del hablante-sujeto (Cuadro 4). Esta función se encuentra otra vez en todas las variedades estudiadas. 


\section{Cuadro 4 \\ El rol semántico del hablante-emisor en las variedades peruanas}

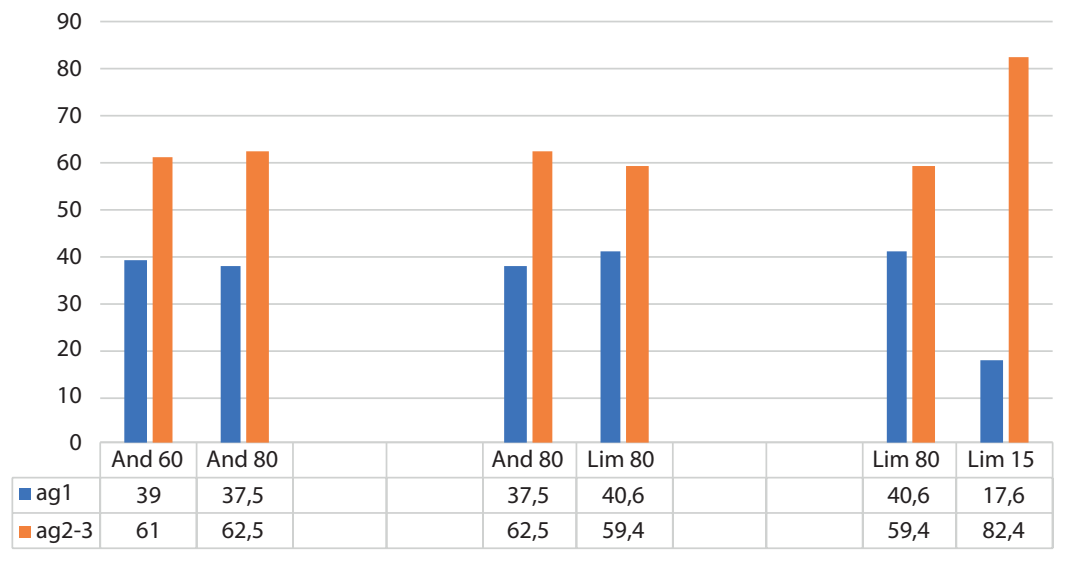

- ag1 =ag2-3

Como es de esperar, la presencia de adverbios temporales es baja en las variedades peruanas (no mostrada aquí). Este factor tiene un promedio de $31 \%$, mientras los valores en las variedades mexicana y peninsular presentadas en Schwenter y Torres Caccoullos (2008) son de 76\% y 77\%, respectivamente. En las variedades peruanas, en cambio, encontramos otras expresiones que son más frecuentes y que pueden proveer una interpretación también temporal si bien no son expresiones adverbiales temporales. Se trata de expresiones plurales (algunas cosas, panes) o colectivos (gente) que llevan a la indeterminación referencial, o incluso de espaciales (en mi tierra) que hacen referencia a 'otro lugar y tiempo anterior'. En el Cuadro 5 se ve cómo la presencia de expresiones no temporales es mayor en los datos andinos del 60 y las variedades limeñas. Otra vez encontramos que los datos Andinos-80 se comportan un poco diferente. Habría que incluir más datos para determinar si es una tendencia con significancia. 


\section{Cuadro 5 \\ La presencia de una expresión temporal de presente en el enunciado según la variedad peruana}

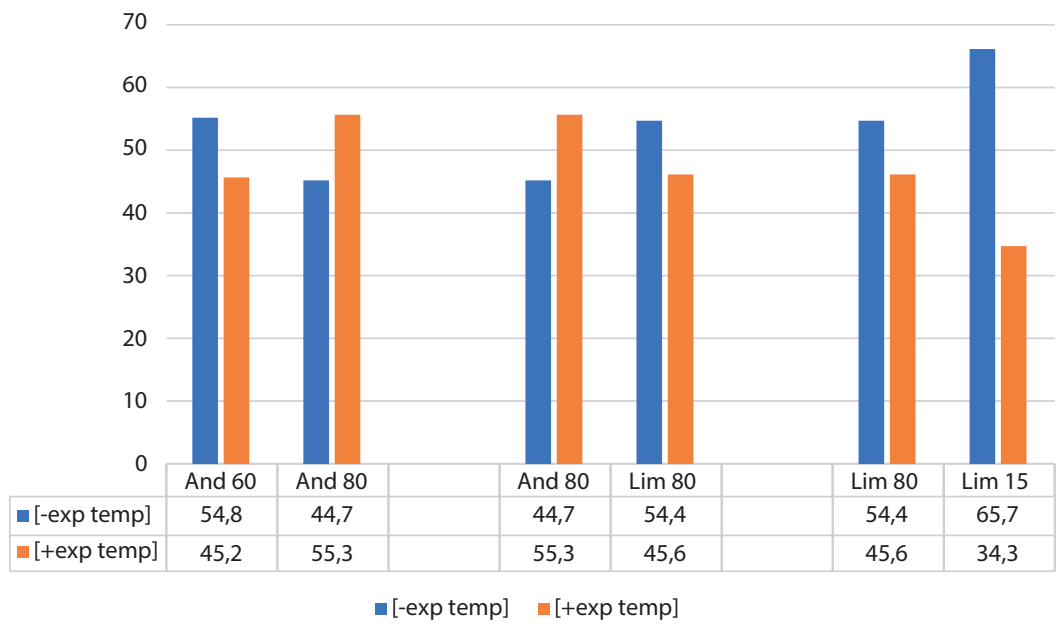

El Cuadro 6 se enfoca en la indeterminación temporal de todas las expresiones, mencionadas antes. Los resultados muestran una tendencia clara en la variedad más moderna (Lima-15) hacia una mayor indeterminación, favorecida por la presencia de expresiones no temporales (Cuadro 5). Los datos sobre la referencia temporal de las pocas expresiones temporales (31\%) también muestran una tendencia hacia expresiones de pasado y no de presente como se espera en otras variedades de español monolingüe, como las variedades mexicana y peninsular que estudian Schwenter y Torres Caccoullos (2008). Los datos corroboran trayectorias de gramaticalización diferentes. Mientras que las otras variedades que aquí se mencionana mantienen el proceso que llamamos tempo-aspectual, la variedad andina peruana representa una trayectoria de gramaticalización pragmático-discursiva (Escobar \& Crespo, 2020). 


\section{Cuadro 6 \\ La indeterminación temporal en el enunciado según la variedad peruana}

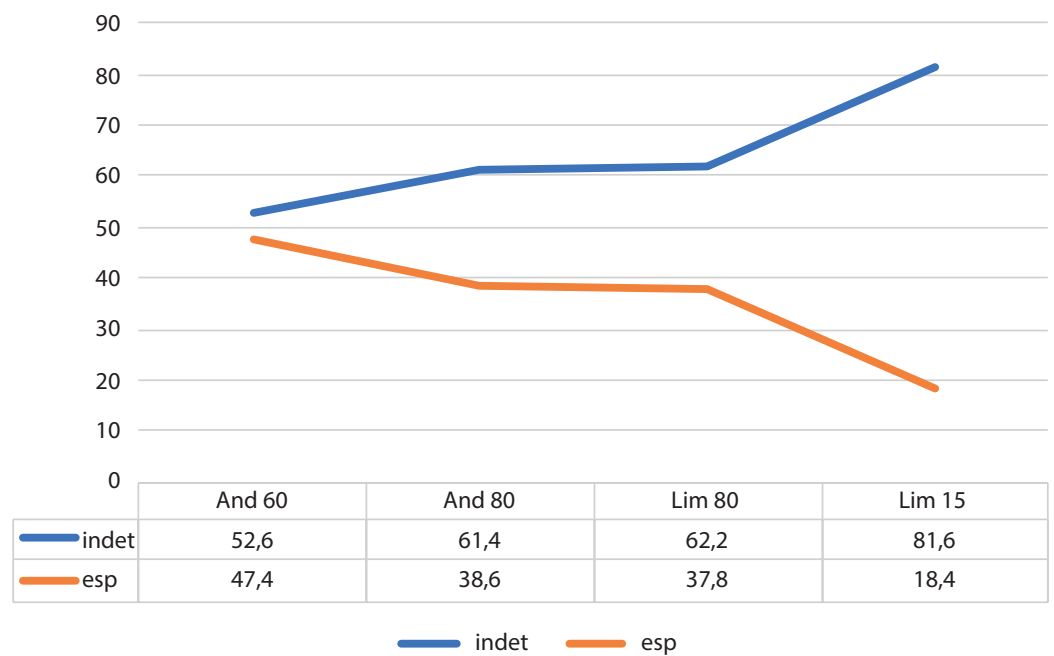

El Cuadro 7 muestra que la telicidad del verbo no es relevante en el favorecimiento del PP-evidencial. Incluso sugeriríamos que la neutralización del Aktionsart es un requisito para la gramaticalización de la subjetividad en el PP-evidencial, como parece ser también la determinación temporal para la gramaticalización en general del $\mathrm{PP}$, como vimos más arriba.

Tanto los verbos télicos (durativo-télico y télico) como los atélicos (de proceso y de estado) pueden aparecer con el PP-evidencial. El evento en sí mismo no se realza con esta forma verbal andina y gramaticalizada, sino la perspectiva del hablante. 


\section{Cuadro 7 \\ El Aktionsart del verbo PP en las variedades peruanas}

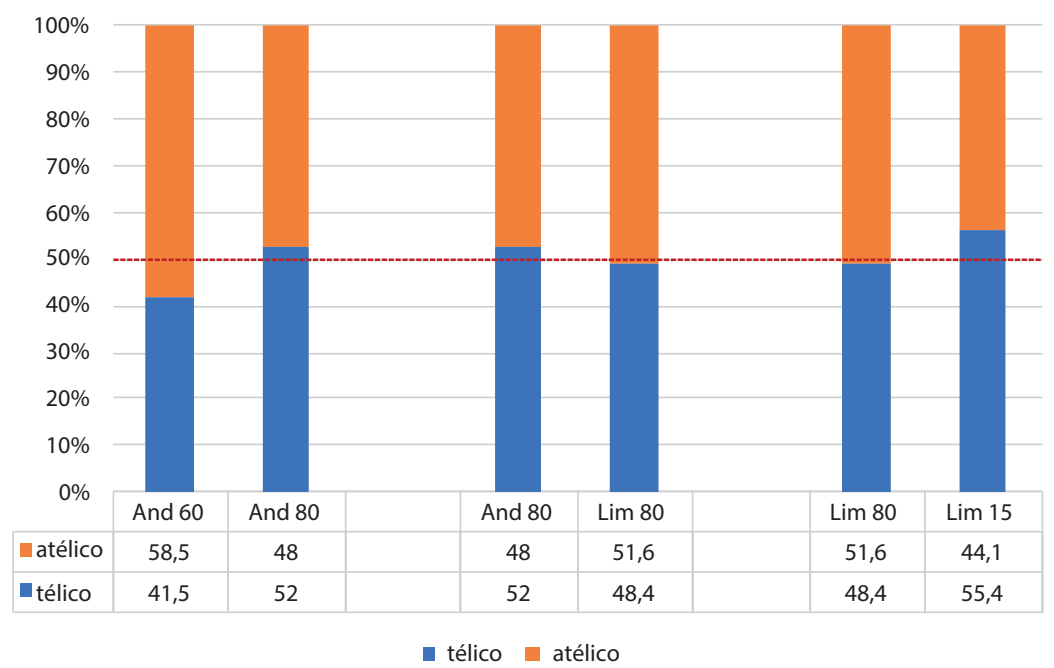

\section{Conclusiones}

Las variables lingüísticas presentadas en este estudio muestran que los factores conectados a temporalidad (la baja presencia de adverbios temporales y la indeterminación temporal) son importantes en el estudio del PP-evidencial, ya que parecen señalar una etapa de gramaticalización más avanzada del PP. La baja presencia de adverbios temporales, además de otras expresiones no temporales que pueden expresar número o espacio y ser interpretadas indirectamente con tiempo o indeterminación, parece ser relevante para una gramaticalización dirigida a la evolución de una función evidencial. Si bien estos factores no aportan un entendimiento de la función evidencial de conocimiento directo que expresa esta forma verbal en 
el español andino peruano, participan de alguna manera en la dinámica del proceso que lleva a la expresión de la función evidencial.

El análisis presentado en la sección 3 sugiere que otros factores lingüísticos conectados al concepto de 'conocimiento directo' sí muestran tendencias al favorecimiento del PP-evidencial. Se trata de (a) la primera persona gramatical, (b) la presencia del hablante en el enunciado en un rol sintáctico, especialmente (c) el rol sintáctico de sujeto, (d) la expresión del pronombre sujeto de primera persona y (e) el rol semántico del sujeto-hablante como experimentante, particularmente, favorecen todos al uso del PP-evidencial andino. Igualmente, los resultados muestran que los factores lingüísticos conectados a la deixis, como (f) la neutralización del tiempo referencial y (g) la indeterminación temporal, son también importantes en la emergencia del PP-evidencial. Los resultados sugieren que la tendencia hacia la atemporalidad da paso a la expresión de funciones pragmáticas, como la subjetividad, que subyace el concepto de 'conocimiento directo'. Al hacer referencia el 'conocimiento directo' al conocimiento del hablante, el foco en el hablante, entonces, realza el vínculo entre la subjetividad y la subcategoría de 'conocimiento directo' que cae bajo evidencialidad.

Los factores lingüísticos conectados al 'conocimiento directo' señalan y confirman que el PP del español andino peruano expresa una función evidencial que no se encuentra en otras variedades de español, especialmente aquellas descritas en las gramáticas normativas. Los siguientes pasos de investigación tendrían que explicar la trayectoria de cómo emergió este significado en estas variedades peruanas a través del tiempo. Así mismo, la documentación de estos usos y de estas trayectorias gramaticales deberán ampliarse a estudios comparativos con otras variedades del español andino, como los bolivianos, los ecuatorianos, los argentinos, los chilenos y otros, 
pues en conjunto ayudarán a documentar la influencia de categorías semánticas del quechua, la lengua minorizada, en la lengua dominante, el español. Igualmente, el entendimiento más refinado del rol que cumplen los factores lingüísticos en la influencia lingüística del quechua en el español ayudarán a entender mejor estos procesos de influencia lingüística que son naturales en las lenguas del mundo, incluyendo aquellas en las situaciones de contacto de lenguas (cf. Heine \& Kuteva, 2005), y de una lengua minorizada a la lengua hegemónica. Por lo tanto, nuestro propósito aquí es también contribuir a la decolonización la perspectiva eurocéntrica y normativa en la lingüística hispánica que ve las variedades andinas de español, como variedades minorizadas social y lingüísticamente. Con este trabajo de documentación empírica, demostramos que los rasgos de las variedades andinas del español representan procesos naturales de cambio lingüístico que se encuentran en las lenguas del mundo y, especialmente, en situaciones de contacto de lenguas, como en la región andina. De esta manera contribuimos a lo que llamamos la lingüística Hispamerindia.

\section{Referencias bibliográficas}

Aikhenvald, A. (2004). Evidentiality. Cambridge: Cambridge University Press. Alcazar, A. (2018). Dizque and other Emergent Evidential Forms in Romance Languages. Aikhenvald, A. (Ed). The Oxford Handbook of Evidentiality. Oxford. Publicación en línea: https://doi.org/10.1093/ oxfordhb/9780198759515.013.35

Álvarez Garriga, D. (2012). Estudio sobre la variación perfecto simple y perfecto compuesto en los discursos presidenciales de Evo Morales: Marcas del contacto lingüístico. Cuadernos de la ALFAL, 4, 30-44.

Andrade Ciudad, L. (2007). Usos de 'dice' en castellano andino. (Tesis doctoral). Pontificia Universidad Católica del Perú.

Anderson, L. (1986). Evidentials, Paths of Change, and Mental Maps: Typologically Regular Asymetries. En Chafe, W. y Nichols, J. (Eds.), Evidentia- 
lity: The Linguistic Coding of Epistemology (pp. 273-312). Norword, NJ: ABLEX Publishing Corporation.

Arellano, R., \& Burgos, D. (2010). Ciudad de los Reyes, de los Chávez, de los Quispe... Lima: Editorial Planeta.

Babel, A. (2009). Dizque, evidentiality, and stance in Valley Spanish. Language in Society, 38, 487-511.

Bergqvist, H. (2017). The role of 'perspective' in epistemic marking. Lingua, 186$187,5-20$.

Boye, K. (2018). Evidentiality: The notion and the term. Aikhenvald, A. (ed.) The Oxford Handbook of Evidentiality. Oxford: Oxford University Press. Publicación en línea: https://doi.org/10.1093/ oxfordhb/9780198759515.013.13

Bustamante, I. (1991). El presente perfecto o pretérito perfecto compuesto en el español quiteño. Lexis 15, 195-231.

Bybee, J., Perkins, R., \& Pagliuca, W. (1994). The evolution of grammar: The grammaticalization of tense, aspect and modality in the languages of the world. Chicago: University of Chicago Press.

Caravedo, R. (1989). El español de Lima. Materiales para el estudio del habla culta. Lima: Pontificia Universidad Católica del Perú.

(1990). Sociolingüistica del español de Lima. Lima: Pontificia Universidad Católica del Perú.

Cerrón Palomino, R. (1987). Lingüistica Quechua. Cuzco: Centro de Estudios Regionales Andinos "Bartolomé de las Casas".

Cerrón Palomino, A. (2018). Null-subject encounter: Variable subject pronoun expression in the Spanish of Quechua-Spanish bilinguals in the Central Peruvian Andes. International Journal of Bilingualism. Recuperado de: https://journals.sagepub.com/doi/10.1177/1367006918763175.

Chafe, W., \& Nichols, J. (1986). Evidentiality: The Linguistic Coding of Epistemology. Norword, NJ: ABLEX Publishing Corporation.

Cornillie, B. (2007). Epistemic and evidential modals: modal grounding a subjectification. En Cornillie, B. (Ed.), Evidentiality and Epistemic Modality in Spanish (Semi)Auxiliaries (pp. 224-252). Berlin, New York: Mouton de Gruyter.

(2017). Conceptual and constructional considerations on the subjectivity of English and Spanish modals. En Athanasiadou, A., Canakis, C. y Cornillie, B. (Eds.), Sujectification. Various Paths to Subjectivity (pp. $177-$ 206). Berlin, New York: De Gruyter Mouton. 
Davidson, B. (1996). 'Pragmatic weight' and subject pronouns: The pragmatic and discourse uses of 'tú' and 'yo' in spoken Madrid Spanish. Journal of Pragmatics, 26, 543-563.

De la Mora, J., \& Maldonado, R. (2015). Dizque: Epistemics blurring evidentials in Mexican Spanish. Journal of Pragmatics, 85, 168-180.

Demonte, V., \& Fernández-Soriano, O. (2013). Evidentials dizque and que in Spanish. Linguistica. Revista do Estudos linguísticos da Universidade do Porto, 8, 211-234.

Dumont, J. (2013). Another look at the Present Perfect in an Andean variety of Spanish: Grammaticalization and Evidentiality in Quiteño Spanish. En Cabrelli Amaro, J. (Ed.) Selected Proceedings of the 16th Hispanic Linguistics Symposium (pp. 279-291). Somerville, MA: Cascadilla Proceedings Project.

Escobar, A. M. (1993). Epistemic Modality in Spanish in Contact with Quechua in Peru. En Smith, E. y Zéphir, F. (Eds.), Proceedings of the Mid-America Linguistics Conference and Conference on Siouan-Caddoan Languages (pp. 137-151). Columbia, Missouri: U. of Missouri.

(1994). Evidential uses in the Spanish of Quechua speakers in Peru. Southwest Journal of Linguistics, 13(1-2), 1-23.

(1997). Contrastive and innovative uses of the present perfect and the preterite in Spanish in contact with Quechua. Hispania, 80, 859-870.

(2014). Los etnolectos y la difusión contrajerárquica: nuevas normas en el español peruano. En Zimmermann, K. (Ed.), Nuevos hispanismos lingüísticos (pp. 259-284). Madrid, Frankfurt: Iberoamericana, Vervuert.

Escobar, A.M. y Crespo, C. (2020). La gramaticalización de la subjetividad en el español andino: el pretérito perfecto compuesto con valor evidencial. En Andrade Ciudad, L. y Sessarego, S. (Eds.). Los castellanos del Perú. Historia, variación y contacto lingüistico. Londres: Routledge.

Faller, M. T. (2002). Semantics and Pragmatics of Evidentials in Cuzco Quechua. (Tesis doctoral). Universidad de Stanford.

(2011). A possible worlds semantics for Cuzco Quechua evidentials. Proceedings of SALT, 20, 660-683.

Floyd, R. (1999). The structure of evidential categories in Wanka Quechua. Dallas, TX: Summer Institute of Linguistics.

García Tesoro, A. I., \& Jang, J. S. (2018). El pretérito perfecto compuesto en el español andino peruano: usos innovadores y extensión a contextos de aoristo. Forma y función, 31(1), 93-123. 
Haboud, M. (1998). Quichua y castellano en los Andes ecuatorianos: los efectos de un contacto prolongado. Quito: Abya-Yala.

Hardman, M. (Ed.) (1981). The Aymara language in its social and cultural context. Gainesville: University of Florida.

(1986). Data-Source Marking in the Jaqi Languages. En Chafe, W. y Nichols, J. (Eds.), Evidentiality: The Linguistic Coding of Epistemology (pp. 113-136). Norword, NJ: ABLEX Publishing Corporation.

Hassler, G. (2015). Evidentiality and the expression of speaker's stance in Romance languages and German. Discourse Studies, 17(2), 182-209.

Heine, B., \& Kuteva, B. (2005). Language contact and grammatical change. Cambridge: Cambridge University Press.

Hennemann, A. (2012). The epistemic and evidential use of Spanish modal adverbs and verbs of cognitive attitude. Folia Linguistica, 46(1), 133-170.

Hintz, D. (2007). Past tense forms and their functions in South Conchucos Quechua. (Tesis doctoral). University of California at Berkeley.

Howe, C. (2006). Cross-dialectal features of the Spanish present perfect: A typological analysis of form and function. (Tesis doctoral). Ohio State University. (2013). The Spanish perfects: Pathways of emergent meaning. Eastbourne, UK: Palgrave Macmillan.

Howe, C., \& Schwenter, S. (2008). Variable constraints on Past Reference in dialects of Spanish. En Westmoreland, M. y Thomas, J.A. (Eds.), Selected Proceedings of the 4th Workshop on Spanish Sociolinguistics (pp. 100-108). Sommerville, MA: Cascadilla Proceedings Project.

Jara Yupanqui, M. (2006). The use of the preterite and the present perfect in the Spanish of Lima. (Tesis doctoral). University of Pittsburgh.

(2013). El Perfecto en el español de Lima: Variación y cambio en situación de contacto lingüistico. Lima: Fondo Editorial de la Pontificia Universidad Católica del Perú.

Klee, C.A. \& Ocampo, A. (1995). The expression of past reference in Spanish narratives of Spanish/Quechua bilingual speakers. En Silva-Corvalán, C. (Ed.), Spanish in contact with other languages (pp. 52-70). Washington D.C.: Georgetown University Press.

Mendoza, J. (1991). El castellano hablado en La Paz: sintaxis divergente. La Paz: Universidad Mayor de San Andrés.

(1992). Gramática castellana con referencia a la variedad hablada en Bolivia. La Paz: Universidad Mayor de San Andrés.

Nuckolls, J. (2014). The Interactional and Cultural Pragmatics of Evidentiality in Pastaza Quichua. En Aikhenvald, A. (Ed.), The Oxford Handbook 
of Evidentiality. Oxford: Oxford University Press. Publicación en línea: https://doi.org/10.1093/oxfordhb/9780198759515.013.10

Nuckolls, J., \& Michael, L. (2014). Evidentials and evidential strategies in interactional and socio-cultural context. En Lev, M. y Nuckolls, J. (Eds.), Evidentials and Evidential Strategies in Social Interaction (pp. 13-20). Ámsterdam: John Benjamins.

Pfänder, S., \& Palacios, A. (2013). Evidencialidad y validación en los pretéritos del español andino ecuatoriano. Círculo de Lingüistica Aplicada a la Comunicación, 54, 65-98.

Rodríguez Louro, C., \& Jara Yupanqui, M. (2011). Otra mirada a los procesos de gramaticalización del Presente Perfecto en español: Perú y Argentina. Studies in Hispanic and Lusophone Linguistics, 4(1), 55-80.

Rodríguez Ramalle, T. (2015). Evidentiality and illative markers in Spanish. Journal of Pragmatics, 85, 200-211.

Sánchez, L. (2004). Functional convergence in the tense, evidentiality, and aspectual systems of the Quechua-Spanish bilinguals. Bilingualism: Language and Cognition, 7, 147-162.

Schumacher de Peña, G. (1975). Observaciones sobre el sistema verbal del español andino. Materiales descriptivos para la enseñanza del castellano como segunda lengua, 63- 104. Lima: Instituto Nacional de Investigación y Desarrollo de la Educación.

(1980). El pasado en español andino de Puno/ Perú. En Bork, D., Greive, A. y Woll, D. (Eds.), Romanica europaea et americana: Festschrift für Harri Meier (pp. 553-58). Bonn: Bouvier Verlang Herbert Grundmann.

Schwenter, S., \& Torres Cacoullos, R. (2008). Defaults and indeterminacy in temporal grammaticalization: The 'perfect' road to perfective. Language Variation and Change, 20(1), 1-39.

Shapero, J. (2017). Does environmental experience shape spatial cognition? Frames of reference among Ancash Quechua speakers (Peru). Cognitive Science, 41(5), 1274-1298.

Stratford, D. (1991). Tense in Altiplano Spanish. Sociolinguistics of the Spanish-speaking world: Iberia, Latin America, United States. Tempe: Bilingual Press.

Traugott, E. C. (1995). Subjectification in Grammaticalization. En Stein, D. y Wright, S. (Eds.), Subjectivity and Subjectivisation: Linguistic Perspectives (pp. 31-54). Cambridge: Cambridge University Press.

(2003). From subjectification to intersubjectification. En Hickey, R. (Ed.), Motives for Language Change (pp. 124-139). Cambridge: Cambridge University Press. 
(2010). Revisiting subjectification and intersubjectification. En Davidse, K., Vandelanotte, L. y Cuyckens, H. (Eds.), Subjectification, Intersubjectification, and Grammaticalization (pp. 29-70). Berlin: De Gruyter Mouton.

Weber, D. (1986). Information Perspective, Profile, and Patterns in Quechua. En Chafe, W. y Nichols, J. (Eds.), Evidentiality: The Linguistic Coding of Epistemology (pp. 137-155). Norword, NJ: ABLEX Publishing Corporation.

Willett, T. (1988). A cross-linguistic survey of the grammaticization of evidentiality. Studies in Language, 12, 51-97.

Van Valin, R. D., \& LaPolla, R. J. (1997). Syntax: Structure, meaning, and function. Cambridge: Cambridge University Press. 